MARCH 1992, PAGES 1-10

\title{
A NOTE ON THE EXISTENCE OF A WAITING TIME FOR A TWO-PHASE STEFAN PROBLEM
}

\author{
By \\ DOMINGO ALBERTO TARZIA (PROMAR, Instituto de Matemática “B. Levi”, Rosario, Argentina) \\ AND \\ CRISTINA VILMA TURNER (Universidad Nacional de Córdoba, Córdoba, Argentina)
}

\begin{abstract}
We consider a slab, represented by the interval $0<x<x_{0}$, at the initial temperature $\theta_{0}=\theta_{0}(x) \geq 0$ (or $\phi_{0}=\phi_{0}(x) \geq 0$ ) having a heat flux $q=q(t)>0$ (or convective boundary condition with a heat transfer coefficient $h$ ) on the left face $x=0$ and a temperature condition $b(t)>0$ on the right face $x=x_{0}\left(x_{0}\right.$ could be also $+\infty$, i.e., a semi-infinite material). We consider the corresponding heat conduction problem and assume that the phase-change temperature is $0^{\circ} \mathrm{C}$.

We prove that certain conditions on the data are necessary or sufficient in order to obtain the existence of a waiting-time at which a phase-change begins.
\end{abstract}

I. Introduction. We consider the following heat conduction problems $\left(0<x_{0} \leq\right.$ $+\infty)$ :

(i) $\rho c \theta_{t}-k \theta_{x x}=0, \quad 0<x<x_{0}, t>0$;

(ii) $\theta(x, 0)=\theta_{0}(x)>0, \quad 0 \leq x \leq x_{0}$;

(iii) $k \theta_{x}(0, t)=q(t), \quad t>0$;

(iv) $\theta\left(x_{0}, t\right)=b(t), \quad t>0$

and

$$
\begin{aligned}
& \text { (i) } \rho c \phi_{t}-k \phi_{x x}=0, \quad 0<x<x_{0}, \quad t>0 \text {; } \\
& \text { (ii) } \phi(x, 0)=\phi_{0}(x)>0, \quad 0 \leq x \leq x_{0} \\
& \text { (iii) } k \phi_{x}(0, t)=h(D+\phi(0, t)), \quad t>0 \\
& \text { (iv) } \phi\left(x_{0}, t\right)=b(t), \quad t>0,
\end{aligned}
$$

where $\rho$ is the density, $k$ is the thermal conductivity, $c$ is the specific heat, $h$ is the convective heat transfer coefficient from a fluid with ambient temperature $-D<0$ flowing across the face $x=0$. The function $b(t)$ represents the temperature at the face $x=x_{0}>0$, and $\theta_{0}$ and $\phi_{0}$ are the initial temperatures for problems (1) and $\left(1^{\prime}\right)$ respectively.

We take, without loss of generality, the phase-change temperature as $0^{\circ} \mathrm{C}$ and replace condition $(1)$ (iv) by $\theta(+\infty, t)=\theta_{0}(+\infty)>0, t>0$ for the case $x_{0}=+\infty$

Received June 1, 1988.

(C) 1992 Brown University 
(idem for Problem $\left(1^{\prime}\right)$ ). We assume that the data satisfy the hypotheses that ensure the existence and uniqueness property of the solution of $(1)$ and $\left(1^{\prime}\right)$.

We consider the following possibilities:

(a) the heat conduction problem is defined for all $t>0$ (waiting-time $t^{*}=+\infty$ );

(b) there exists a time $t^{*}<+\infty$ such that another phase (i.e., the solid phase) appears for $t \geq t^{*}$ (waiting-time $0 \leq t^{*}<+\infty$ ) and then we have a two-phase Stefan problem for $t>t^{*}$. In this case, there exists a free boundary $x=s(t)$, which separates the liquid and solid phases with $s\left(t^{*}\right)=0$.

We will separate the cases waiting-time $t^{*}=0$ (i.e., there exists an instantaneous change of phase) and $0<t^{*} \leq+\infty$. These possibilities depend on the data $\theta_{0}, q$, $b$ for Problem (1) and the data $\phi_{0}, h, b$ for Problem $\left(1^{\prime}\right)$. We try to clarify this dependence by finding necessary or sufficient conditions on $\theta_{0}, q, b$ and $\phi_{0}, h$, $b$ in order to have the different possibilities.

In $[5,8,9]$ the one-phase Stefan problem with prescribed flux or convective boundary condition at $x=0$ is studied.

This paper was motivated by $[10,12,13]$ (see also [14]) and the term waitingtime was motivated by its correspondence to the term as used in the porous medium equation (see, for instance [1]).

In Sec. II we analyse problem (1) with a flux boundary condition at $x=0$ and in Sec. III we study the problem $\left(1^{\prime}\right)$ with a convective boundary condition at $x=0$.

II. On some conduction problems with a flux boundary condition. We consider the following properties for the problem (1).

THEOREM 1. If the data $q=q(t), \theta_{0}=\theta_{0}(x)$, and $b=b(t)$ verify conditions

(i) $0<q(t) \leq q_{0}, \quad 0<t \leq t_{0}$ with $t_{0}>0$;

(ii) $\theta_{0}^{\prime}(x) \geq 0 \quad$ and $\quad \beta_{1} \geq \theta_{0}(x) \geq \beta_{0}>0, \quad 0 \leq x \leq x_{0}$ with $\beta_{0} \leq \beta_{1}$;

(iii) $b(t) \geq \beta_{1}$ and $\dot{b}(t) \geq 0, t>0$;

then there exists a waiting-time $t^{*}>0$ for problem (1), (i.e., another phase could appear at $t \geq t^{*}$ ), where $t^{*}$ verifies the following inequality:

$$
t^{*} \geq \operatorname{Min}\left(t_{0}, t_{0}^{*}\right), \quad \text { where } t_{0}^{*}=\pi k \rho c \beta_{0}^{2} / 4 q_{0}^{2} .
$$

Proof. It is sufficient to prove that $\theta(x, t) \geq 0$ for $0 \leq x \leq x_{0}$ and $0 \leq t \leq t_{0}^{*}$. For the semi-infinite material $x>0$, with the same thermal coefficients, we consider the following two problems:

$$
\begin{aligned}
\rho c T_{t}-k T_{x x} & =0, \quad x>0, \quad 0<t<t_{0} ; \\
k T_{X}(0, t) & =q(t), \quad t>0 ; \\
T(x, 0) & =T_{0}(x), \quad x \geq 0,
\end{aligned}
$$

with

$$
T_{0}(x)= \begin{cases}\theta_{0}(x), & 0 \leq x \leq x_{0}, \\ \theta_{0}\left(x_{0}\right), & x>x_{0},\end{cases}
$$


and

$$
\begin{aligned}
\rho c V_{t}-k V_{x x} & =0, \quad x>0, \quad t>0 ; \\
k V_{x}(0, t) & =q_{0}>0, \quad t>0 ; \\
V(x, 0) & =\beta_{0}>0, \quad x \geq 0,
\end{aligned}
$$

whose solutions are given respectively by $[3,4]$

$$
T(x, t)=\int_{0}^{+\infty} N(x, t ; \xi, 0) T_{0}(\xi) d \xi-\frac{2 a}{k} \int_{0}^{t} K(x, t ; 0, \tau) q(\tau) d \tau,
$$

and

$$
V(x, t)=\beta_{0}-\frac{2 q_{0} a}{k} \sqrt{t} i \operatorname{erfc}\left(\frac{x}{2 a \sqrt{t}}\right),
$$

where

$$
\begin{gathered}
a=\left(\frac{k}{\rho c}\right)^{1 / 2} ; \quad K(x, t ; \xi, \tau)=\frac{\exp \left(-(x-\xi)^{2} / 4 a^{2}(t-\tau)\right)}{2 a \sqrt{\pi(t-\tau)}} ; \\
N(x, t ; \xi, \tau)=K(x, t ; \xi, \tau)+K(-x, t ; \xi, \tau) ; \quad \operatorname{erf}(x)=\frac{2}{\sqrt{\pi}} \int_{0}^{x} \exp \left(-t^{2}\right) d t \\
\operatorname{erfc}(x)=1-\operatorname{erf}(x) ; \quad i \operatorname{erfc}(x)=\frac{\exp \left(-x^{2}\right)}{\sqrt{\pi}}-x \operatorname{erfc}(x) .
\end{gathered}
$$

By the maximum principle $[6,7]$ we obtain

$$
\begin{aligned}
& V(x, t) \leq T(x, t), \quad x \geq 0,0 \leq t \leq t_{0}, \\
& T(x, t) \leq \theta(x, t), \quad 0 \leq x \leq x_{0}, 0 \leq t \leq t_{0},
\end{aligned}
$$

because $T\left(x_{0}, t\right) \leq \beta_{1} \leq b(t)$ for $0 \leq t \leq t_{0}$.

Let $W$ be the function $W=\theta_{x}$, which satisfies the following heat conduction problem:

$$
\begin{aligned}
& \rho c W_{t}-k W_{x x}=0, \quad 0<x<x_{0}, t>0 ; \\
& W(x, 0)=\theta_{0}^{\prime}(x), \quad 0 \leq x \leq x_{0} ; \\
& W(0, t)=\frac{q(t)}{k}, \quad W_{x}\left(x_{0}, t\right)=\frac{\rho c}{k} \dot{b}(t), t>0 .
\end{aligned}
$$

By the maximum principle we have $W=\theta_{x} \geq 0$ for $0 \leq x \leq x_{0}, t \geq 0$. Then, we deduce that

$$
\theta(x, t) \geq \theta(0, t) \geq V(0, t)=\beta_{0}-\frac{2 q_{0} a}{k} \sqrt{\frac{t}{\pi}} \geq 0 \quad \text { for } 0 \leq t \leq t_{0}^{*},
$$

where $t_{0}^{*}$ is defined by (3) proving our assertion.

REMARK 1 . When the data verify conditions (2), problem (1) represents a heat conduction problem for the initial phase (in our case, the liquid phase) for $t \leq t^{*}$.

Remark 2. We can see that $t_{0}^{*}$ does not depend on the length of the slab $x_{0}>0$. Corollary 2. Under the hypotheses (2)(ii),(iii), a necessary condition in order to have an instantaneous change of phase (i.e., $t^{*}=0$ ) for problem (1) is given by

$$
q\left(0^{+}\right)=+\infty \text {. }
$$


REMARK 3. If we consider the case

$$
\begin{aligned}
& x_{0}=+\infty, \quad \theta_{0}(x) \geq \beta_{0}>0, \quad \forall x \geq 0, \\
& q(t) \leq q_{0}(t)=\frac{\beta_{0} k}{a \sqrt{\pi} t}, \quad \forall t>0,
\end{aligned}
$$

then problem (1) is a heat conduction problem for the liquid phase for all $t>0$ i.e., there is not a phase-change process for any $t>0$ because we have

$$
\theta(x, t) \geq \theta q_{0}(x, t), \quad x \geq 0, t>0,
$$

where $\theta q_{0}$ is the solution of (1) with data: heat flux $q_{0}(t)$ at $x=0, x_{0}=+\infty$, anc initial temperature $\beta_{0}$. It is given by [12]

$$
\theta q_{0}(x, t)=\beta_{0} \operatorname{erf}\left(\frac{x}{2 a \sqrt{t}}\right) \geq 0, \quad x \geq 0, t>0 .
$$

Moreover, the particular case

$$
q(t)=\frac{\beta_{0} k}{a \sqrt{\pi t}}=q_{0}(t), \quad t>0,
$$

shows us that condition (13) is not sufficient in order to have an instantaneous change of phase for problem (1).

REMARK 4. If $x_{0}=+\infty$ and $\theta_{0}(x) \geq \beta_{0}>0$ for $x \geq 0$, then a necessary condition for problem (1) to have an instantaneous change of phase (i.e., the waiting. time is $t^{*}=0$ ) is for there to exist a $t_{0}>0$ such that

$$
q\left(t_{0}\right)>\frac{\beta_{0} k}{a \sqrt{\pi t_{0}}} .
$$

THEOREM 3. If the data verify the conditions

$$
\begin{array}{ll}
x_{0}=+\infty ; & 0 \leq \theta_{0}(x) \leq \beta_{1} \text { for } x \geq 0, \\
q(t) \geq \frac{q_{0}}{t^{\beta}}, & 0<t<1, \text { with } q_{0}>0 \text { and } \frac{1}{2}<\beta<1,
\end{array}
$$

then an instantaneous phase-change occurs, that is, the waiting-time is $t^{*}=0$.

Proof. Let $U=U(x, t)$ be the solution of the following heat conduction problem:

$$
\begin{array}{rlrl}
\rho c U_{t}-k U_{x x} & =0, & x>0, \quad t>0 ; \\
U(x, 0) & =\beta_{1}, & & x \geq 0 ; \\
k U_{x}(0, t) & =\frac{q_{0}}{t^{\beta}}, & & t>0,
\end{array}
$$

which is given by [3]

$$
U(x, t)=\beta_{1}-\frac{2 a q_{0}}{k} \int_{0}^{t} \frac{K(x, t ; 0, \tau)}{\tau^{\beta}} d \tau .
$$

By using the maximum principle we have that $\theta(x, t) \leq U(x, t)$ for $x \geq 0, t>0$. Therefore, we obtain

$$
\theta(0, t) \leq U(0, t) \leq \beta_{1}-\frac{a q_{0}}{k \sqrt{\pi}} \int_{0}^{t} \frac{d \tau}{\tau^{\beta} \sqrt{t-\tau}}
$$


and, for $0<\varepsilon=t / 2<t<1$,

$$
\int_{0}^{t} \frac{d \tau}{\tau^{\beta} \sqrt{t-\tau}} \geq \int_{0}^{\varepsilon} \frac{d \tau}{\varepsilon^{\beta} \sqrt{t-\tau}}+\int_{\varepsilon}^{t} \frac{d \tau}{\tau^{\beta} \sqrt{t-\varepsilon}}=\mathrm{C}_{\beta}\left(\frac{2}{t}\right)^{\beta-1 / 2}
$$

Moreover, the temperature on the fixed face $x=0$ verifies the inequality

$$
\theta(0, t) \leq \beta_{1}-\frac{a q_{0}}{k \sqrt{\pi}} \mathrm{C}_{\beta}\left(\frac{2}{t}\right)^{\beta-1 / 2}<0,
$$

for all $t<\min \left(1, t_{\beta}\right)$, where

$$
\begin{aligned}
t_{\beta} & =2\left(\frac{a q_{0}}{k \beta_{1} \sqrt{\pi}} \mathrm{C}_{\beta}\right)^{1 /(\beta-1 / 2)}>0, \\
\mathrm{C}_{\beta} & =\frac{1}{1-\beta}\left[2^{1-\beta}-1+2(1-\beta)(\sqrt{2}-1)\right]>0,
\end{aligned}
$$

that is, the thesis is achieved.

REMARK 5. If we consider the density jump under the phase of change, that is, $\rho_{1} \neq \rho_{2}$, and the data verify the conditions

$$
\begin{aligned}
x_{0} & =+\infty ; \quad 0 \leq \theta_{0}(x) \leq \beta_{1} \text { for } x \geq 0, \\
q(t) & \geq \frac{q_{0}}{\sqrt{t}} \text { for } t>0 \text { with } q_{0}>\frac{\beta_{1} k_{2}}{a_{2} \sqrt{\pi}},
\end{aligned}
$$

where $k_{i}, c_{i}, \rho_{i}, a_{i}=\left(k_{i} / \rho_{i} c_{i}\right)^{1 / 2}$ are the corresponding thermal coefficients for the phase $i\left(i=2\right.$ : liquid phase, $i=1$ : solid phase), then the temperature $\theta=\theta_{q, \theta_{0}}$, solution of problem (1), verifies the inequality $\theta_{q, \theta_{0}}(x, t) \leq T_{q_{0}, \beta_{1}}(x, t), x \geq 0$, $t>0$, where $T_{q_{0}, \beta_{1}}$ is the solution of (1) with initial constant temperature $\beta_{1}$ and a flux condition of type $q_{0} / \sqrt{t}$ on $x=0$. Therefore, we obtain [2]

$$
\theta_{q, \theta_{0}}(0, t) \leq T_{q_{0}, \beta_{1}}(0, t)=\beta_{1}-\frac{q_{0} a_{2} \sqrt{\pi}}{k_{2}}<0, \quad t>0,
$$

that is, the waiting-time is $t^{*}=0$ (i.e., we have an instantaneous two-phase Stefan problem) for data $q$ and $\theta_{0}$. Moreover, its free boundary $x=s_{q, \theta_{0}}(t)$ verifies $s_{q, \theta_{0}}(0)=0$ and it is characterized by $\theta_{q, \theta_{0}}\left(s_{q, \theta_{0}}(t), t\right)=0$ for all $t>0$. by [2]

The free boundary $x=s_{q_{0}, \beta_{1}}(t)$ corresponding to the temperature $T_{q_{0}, \beta_{1}}$ is given

$$
s_{q_{0}, \beta_{1}}=2 \omega \sqrt{t}
$$

where $\omega$ is the unique solution of the equation

$$
F_{0}(x)=x, \quad x>0
$$

with

$$
F_{0}(x)=\frac{q_{0}}{h \rho_{1}} \exp \left(\frac{-x^{2}}{a_{1}^{2}}\right)-\frac{k_{2} \beta_{1}}{h \rho_{1} a_{2} \sqrt{\pi}} \frac{\exp \left(-x^{2} / a_{2}\right)}{\operatorname{erfc}\left(x / a_{2}\right)},
$$

where $h>0$ is the latent heat. Owing to

$$
\theta_{q, \theta_{0}}\left(s_{q_{0}, \beta_{1}}(t), t\right) \leq T_{q_{0}, \beta_{1}}\left(s_{q_{0}, \beta_{1}}(t), t\right)=0, \quad \text { for all } t>0,
$$


it follows that

$$
s_{q, \theta_{0}}(t) \geq s_{q_{0}, \beta_{1}}(t)=2 \omega \sqrt{t}, \quad t>0
$$

From now on we consider the particular case of constant temperature $b(t)=b>$ $0, t>0$ at $x=x_{0}$ and constant heat flux $q(t)=q>0, t>0$ at $x=0$ for problem (1). The steady-state solution is given by

$$
\theta_{\infty}(x)=\frac{q}{k}\left(x-x_{0}\right)+b,
$$

and a necessary and sufficient condition in order to have a two-phase steady-state Stefan problem is given by

$$
q>k b / x_{0},
$$

where $k$ is the thermal conductivity of the liquid phase [11]. (See [13, 14] for the general steady-state case for an $n$-dimensional domain).

Using the fact that $\theta=\theta(x, t)$, the solution of problem (1) with data $q>0$ and $b>0$, converges to $\theta_{\infty}=\theta_{\infty}(x)$ when $t$ goes to $+\infty$ [6], for any initial temperature $\theta_{0}=\theta_{0}(x)$, we can formulate the following problem: Find the relation between the heat flux $q>0$ on $x=0$ and a time $t_{1}$ such that another phase appears for $t \geq t_{1}$, and then we can reformulate problem (1) in a two-phase Stefan problem for $t \geq t_{1}$.

We obtain the following result.

THEOREM 4. Suppose the initial temperature verifies the conditions $b \geq \theta_{0} \geq 0$ in $\left[0, x_{0}\right]$ and $\theta_{0}\left(x_{0}\right)=b$. If the time $t_{1}>0$ and the constant heat flux $q>0$ verify the inequality

$$
q>\frac{b k}{x_{0}\left(1-\exp \left(-\alpha \pi^{2} t_{1} / 4 x_{0}^{2}\right)\right)}, \quad \alpha=\frac{k}{\rho c},
$$

then another phase (the solid phase) appears for $t \geq t_{1}$. Moreover, $\theta(0, t)<0$ for all $t \geq t_{1}$ and the free boundary $x=s(t)$ begins at a point $\left(0, t^{\prime}\right)$ with $0 \leq t^{\prime}<t_{1}$.

Proof. The temperature $\theta(x, t)$ is given by

$$
\theta(x, t)=\theta_{\infty}(x)+\sum_{n=0}^{\infty} \mathrm{C}_{n} \cos \left(\sqrt{\lambda_{n}} x\right) \exp \left(-\alpha t \lambda_{n}\right)
$$

where

$$
\begin{aligned}
\lambda_{n} & =\left(n+\frac{1}{2}\right)^{2} \frac{\pi^{2}}{x_{0}^{2}}, \quad n=0,1,2, \ldots \\
\mathrm{C}_{n} & =\frac{2}{x_{0}} \int_{0}^{x_{6}}\left[\theta_{0}(x)-\theta_{\infty}(x)\right] \cos \left(\sqrt{\lambda_{n}} x\right) d x .
\end{aligned}
$$

Therefore, the temperature at $x=0$ is given by

$$
\theta(0, t)=b-\frac{q x_{0}}{k}(1+S(t))+S_{0}(t)
$$

with

$$
S(t)=\frac{2}{x_{0}^{2}} \sum_{n=0}^{\infty} \exp \left(-\alpha t \lambda_{n}\right) \int_{0}^{x_{0}}\left(x-x_{0}\right) \cos \left(\sqrt{\lambda_{n} x}\right) d x=-\frac{2}{\pi^{2}} \sum_{n=0}^{+\infty} \frac{\exp \left(-\alpha t \lambda_{n}\right)}{\left(n+\frac{1}{2}\right)^{2}}
$$


and

$$
S_{0}(t)=\frac{2}{x_{0}} \sum_{n=0}^{+\infty} \exp \left(-\alpha t \lambda_{n}\right) \int_{0}^{x_{0}}\left(\theta_{0}(x)-b\right) \cos \left(\sqrt{\lambda_{n}} x\right) d x .
$$

We get that $S_{0}(t)=v(0, t) \leq 0$ for all $t \geq 0$ because of the maximum principle, where the function $v=v(x, t) \leq 0$ is the solution of the problem

$$
\begin{array}{rlrl}
\rho c v_{t}-k v_{x x} & =0, & & 0<x<x_{0}, \quad t>0 ; \\
v_{x}(0, t) & =0, \quad t>0 ; \\
v\left(x_{0}, t\right) & =0, \quad t>0 ; \\
v(x, 0) & =\theta_{0}(x)-b \leq 0, \quad 0 \leq x \leq x_{0} .
\end{array}
$$

By some manipulations, it follows that

$$
0<|S(t)|=-S(t) \leq \exp \left[-\frac{\alpha \pi^{2} t}{4 x_{0}^{2}}\right]<1 \quad \text { for all } t \geq t_{1} .
$$

Therefore, if $q$ and $t_{1}$ verify (35), we obtain

$$
\theta(0, t) \leq b-\frac{q x_{0}}{k}(1+S(t))<0 \text { for all } t \geq t_{1}
$$

i.e., the thesis is achieved.

REMARK 6. If $\theta_{0}(x)=b$ in $\left[0, x_{0}\right]$, we deduce $S_{0}(t)=0$ for $t>0$. We remark that inequality (35) was obtained for this particular initial temperature because $S_{0}(x) \leq 0$ in $(0,+\infty)$ for $\theta_{0}(x) \leq b$ in $\left[0, x_{0}\right]$.

Corollary 5. If we consider the $t, q$ plane and define the following set

$$
Q=\{(t, q) \mid q>f(t), t>0\}, \quad f(t)=\frac{b k}{x_{0}\left[1-\exp \left(-\alpha \pi^{2} t / 4 x_{0}^{2}\right)\right]},
$$

then we have a two-phase problem for all $(t, q) \in Q$.

III. On some conduction problems with a convective boundary condition. Now we consider the same kind of techniques used in Sec. 2 for problem $\left(1^{\prime}\right)$ corresponding to a heat conduction problem with a convective boundary condition at $x=0$.

TheOREM 6. If the data $\phi_{0}=\phi_{0}(x), b=b(t)$, and $D$ verify the conditions

$$
\begin{aligned}
& \text { (i) } \phi_{0}^{\prime}(x) \geq 0 \text { and } \beta_{1} \geq \phi_{0}(x) \geq \beta_{0}>0, \quad 0 \leq x \leq x_{0} \text {; } \\
& \text { (ii) } b(t) \geq \beta_{1} \text { and } \dot{b} \geq 0, \quad t>0 \\
& \text { (iii) } D>0
\end{aligned}
$$

then there exists a waiting-time $t^{*}>0$ for problem $\left(1^{\prime}\right)$, where $t^{*}$ verifies the inequality

$$
t^{*} \geq t_{1}^{*}, \quad \text { where } t_{1}^{*}=\frac{k c \rho}{h^{2}}\left(F^{-1}\left(1+\frac{\beta_{0}}{D}\right)\right)^{2},
$$

where $F^{-1}$ is the inverse function of

$$
F(x)=\frac{\exp \left(-x^{2}\right)}{\operatorname{erfc}(x)}, \quad x>0
$$


Proof. By using the maximum principle we get that

$$
\begin{aligned}
\phi_{x}(x, t) & \geq 0, \quad 0 \leq x \leq x_{0}, \quad t \geq 0, \\
\phi(x, t) & \geq z(x, t), \quad 0 \leq x \leq x_{0}, t \geq 0,
\end{aligned}
$$

where $z=z(x, t)$ is the solution of the following heat conduction problem:

$$
\begin{aligned}
& \text { (i) } \rho c z_{t}-k z_{x x}=0, \quad x>0, t>0 ; \\
& \text { (ii) } z(x, 0)=\beta_{0}, \quad x>0 ; \\
& \text { (iii) } k z_{k}(0, t)=h(D+z(0, t)), \quad t>0,
\end{aligned}
$$

which is given by $\left(a^{2}=k / \rho c\right)$ :

$$
\begin{gathered}
z(x, t)=\left(\beta_{0}+D\right)\left[\operatorname{erfc}\left(\frac{x}{2 a \sqrt{t}}\right)+\exp \left(\frac{h x}{k}+\eta^{2}\right)\right] \operatorname{erfc}\left(\frac{x}{2 a \sqrt{t}}+\eta\right), \\
x \geq 0, t \geq 0,
\end{gathered}
$$

where

$$
\eta=\frac{h a \sqrt{t}}{k}
$$

Taking into account (47) and (49) we get

$$
\phi(x, t) \geq \phi(0, t) \geq z(0, t)=-D+\left(\beta_{0}+D\right) \exp \left(\eta^{2}\right) \operatorname{erfc}(\eta) \geq 0, \quad t \leq t_{1}^{*},
$$

because the function $F(x)$ verifies the conditions

$$
F(0)=1, \quad F(+\infty)=+\infty, \quad F^{\prime}>0 \quad \text { in } R^{+} .
$$

REMARK 7. We can see that $t_{1}^{*}$ does not depend on the length of the slab $x_{0}>0$.

From now on we consider the particular case of constant temperature $b(t)=b>$ $0, t>0$ at $x=x_{0}$ for problem $\left(1^{\prime}\right)$. The corresponding steady-state solution is given by

$$
\phi_{\infty}(x)=b-\frac{h(D+b)}{k+h x_{0}}\left(x_{0}-x\right), \quad 0 \leq x \leq x_{0},
$$

and a necessary and sufficient condition, in order to have a two-phase steady-state Stefan problem is given by

$$
h>\frac{k b}{D x_{0}} .
$$

We consider the following problem related to problem $\left(1^{\prime}\right)$ : Find the relation between the heat transfer coefficient $h$ and a time $t_{2}$ such that another phase appears for $t \geq t_{2}$, and then we can reformulate problem $\left(1^{\prime}\right)$ in a two-phase Stefan problem for $t \geq t_{2}$. We obtain the following result.

THEOREM 7. Suppose the initial temperature verifies the conditions $b \geq \theta_{0} \geq 0$ in $\left[0, x_{0}\right]$ and $\theta_{0}\left(x_{0}\right)=b$. If the time $t_{2}>0$ and the constant heat transfer coefficient $h>0$ verify the inequality

$$
h>g\left(t_{2}\right) \text {, }
$$


then another phase (the solid phase) appears for $t \geq t_{2}$, where the function $g=g(t)$ is defined implicitly by the equation

$$
\psi(t, g(t))=0, \quad t>0,
$$

with

$$
\begin{aligned}
& \psi(t, h)=-D+\frac{k(D+b)}{k+h x_{0}}+\frac{2 k(D+b)}{h x_{0}} \gamma(t), \quad t>0, h>0, \\
& \gamma(t)=\sum_{n=1}^{\infty} \exp \left[-\frac{(2 n-1)^{2} \pi^{2} a^{2} t}{x_{0}^{2}}\right], \quad t>0 .
\end{aligned}
$$

Proof. The solution of problem $\left(1^{\prime}\right)$ is given by

$$
\begin{gathered}
\phi(x, t)=\phi_{\infty}(x)+\sum_{n=1}^{\infty} B_{n} \exp \left(-\mu_{n}^{2} a^{2} t\right)\left[\operatorname{Sin}\left(\mu_{n} x\right)+\frac{k \mu_{n}}{h} \operatorname{Cos}\left(\mu_{n} x\right)\right], \\
0 \leq x \leq x_{0}, t>0,
\end{gathered}
$$

where

$$
B_{n}=\frac{2}{x_{0}} \int_{0}^{x_{0}}\left[\phi_{0}(x)-\phi_{\infty}(x)\right]\left[\operatorname{Sin}\left(\mu_{n} x\right)+\frac{k \mu_{n}}{h} \operatorname{Cos}\left(\mu_{n} x\right)\right] d x,
$$

and $\mu_{n}=\omega_{n} / x_{0}$, where $\omega_{n}$ is the $n$th root of the eigenvalue equation

$$
\operatorname{tg}(\omega)=-\frac{k}{h x_{0}} \omega, \quad \omega>0
$$

Moreover, we get that

$$
(2 n-1) \frac{\pi}{2}<\omega_{n}<n \pi, \quad n \in \mathbb{N} .
$$

After some manipulation, we deduce that the temperature at $x=0$ is bounded by

$$
\phi(0, t) \leq \psi(t, h), \quad t>0,
$$

where the function $\psi$ has been defined before.

We notice that the function $g=g(t)$ is well defined since the functions $\gamma=\gamma(t)$ and $\psi=\psi(t, h)$ satisfy the properties

$$
\gamma\left(0^{+}\right)=+\infty, \quad \gamma(+\infty)=0, \quad \gamma^{\prime}(t)<0, \forall t>0,
$$

(a) $\psi\left(t, 0^{+}\right)=+\infty, \quad \psi(t,+\infty)=-D<0, \quad t>0$;

(b) $\frac{\partial \psi}{\partial h}(t, h)<0, \quad \frac{\partial \psi}{\partial t}(t, h)<0, \quad t>0, h>0$.

Therefore, the function $g=g(t)$ satisfies the conditions

$$
g\left(0^{+}\right)=+\infty, \quad g(+\infty)=\frac{k b}{D x_{0}}, \quad g^{\prime}(t)<0, \forall t>0 .
$$

By using the inequality (65) we get the thesis. 
Corollary 8 . We consider in the plane $t, h$ the following set:

$$
R_{2}=\{(t, h) \mid h>g(t), t>0\} ;
$$

then we have a two-phase problem for all $(t, h) \in R_{2}$.

REMARK 8. If the initial temperature is given by $\phi_{0}(x)=b>0$ in $\left[0, x_{0}\right]$, then we have a heat conduction problem for the initial phase for all $(t, h) \in R_{1}$, where

$$
R_{1}=\left\{(t, h) \mid 0<h<\operatorname{Max}\left(\frac{k b}{D x_{0}}, F^{-1}\left(1+\frac{b}{D}\right) \sqrt{\frac{k \rho c}{t}}\right), t>0\right\} .
$$

Acknowledgment. This paper has been partially sponsored by the Project "Problemas de Frontera Libre de la Física-Matemática" (CONICET-UNR, Argentina).

\section{REFERENCES}

[1] D. G. Aronson, The porous medium equation, Nonlinear Diffusion Problems (A. Fasano and M. Primicerio, eds.), Lecture Notes in Math., Vol. 1224, Springer-Verlag, Berlin, 1986, pp. 1-46

[2] A. B. Bancora and D. A. Tarzia, On the Neumann solution for the two-phase Stefan problem including the density jump at the free boundary, Lat. Am. J. Heat Mass Transfer 9, 215-222 (1985)

[3] J. R. Cannon, The One-dimensional Heat Equation, Addison-Wesley, Menlo Park, California, 1967

[4] H. S. Carslaw and J. C. Jaeger, Conduction of Heat in Solids, Clarendon Press, Oxford, 1959

[5] A. Fasano and M. Primicerio, General free boundary problems for the heat equation. I, J. Math. Anal. Appl. 57, 694-723 (1977)

[6] A. Friedman, Partial Differential Equations of Parabolic Type, Prentice-Hall, Englewood Cliffs, 1964

[7] M. H. Protter and H. F. Weinberger, Maximum Principles in Differential Equations, Prentice-Hall, Englewood Cliffs, 1967

[8] B. Sherman, General one-phase Stefan problems and free boundary problems for the heat equation with Cauchy data prescribed on the free boundary, SIAM J. Appl. Math. 20, 555-570 (1971)

[9] A. D. Solomon, V. Alexiades, and D. G. Wilson, The Stefan problem with a convective boundary condition, Quart. Appl. Math. 40, 203-217 (1982)

[10] A. D. Solomon, D. G. Wilson, and V. Alexiades, Explicit solutions to change problems, Quart. Appl. Math. 41, 237-243 (1983)

[11] D. A. Tarzia, Sobre el caso estacionario del problema de Stefan a dos fases, Math. Notae 28, 73-89 (1980)

[12] D. A. Tarzia, An inequality for the coefficient $\sigma$ of the free boundary $s(t)=2 \sigma \sqrt{t}$ of the Neumann solution for the two-phase Stefan problem, Quart. Appl. Math. 39, 491-497 (1981-82)

[13] D. A. Tarzia, An inequality for the constant heat flux to obtain a steady-state two-phase Stefan problem, Engineering Analysis 5, 177-181 (1988). See also On heat flux in materials on free boundary problems: Theory and applications, Irsee/Bavaria, 11-20 June 1987, Res. Notes in Math., No. 186, Pitman, London, 1990, pp. 703-709.

[14] D. A. Tarzia, The two-phase Stefan problem and some related conduction problems, Reuniòes em Matemática Aplicada e Computacào Científica, Vol. 5, SBMAC-Soc. Brasileira Mat. Apl. Comput., Gramado, 1987 